\title{
Norovirus gastroenteritis outbreak transmitted by food and vomit in a high school
}

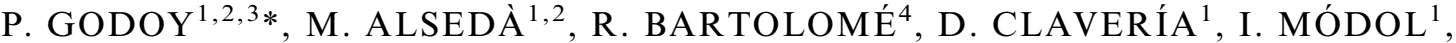 \\ P. BACH ${ }^{1}$, G. MIRADA ${ }^{1}$ AND À. DOMÍNGUEZ ${ }^{3,5}$, on behalf of Working Group for the \\ Study of Outbreaks of Acute Gastroenteritis in Catalonia $\dagger$
}

${ }^{1}$ Departament de Salut, Generalitat de Catalunya, Lleida, Spain

${ }^{2}$ Institut de Recerca Biomédica de Lleida (IRBLleida), Universitat de Lleida, Lleida, Spain

${ }^{3}$ CIBER Epidemiología y Salud Pública (CIBERESP), Madrid, Spain

${ }^{4}$ Hospital de la Vall d'Hebrón, Barcelona, Spain

${ }^{5}$ Departament de Salut Pública, Universitat de Barcelona, Barcelona, Spain

Received 24 September 2015; Final revision 8 December 2015; Accepted 16 December 2015; first published online 13 January 2016

\section{SUMMARY}

We investigated an outbreak of norovirus that affected students and teachers of a high school in Lleida, Spain through various transmission mechanisms. A case-control epidemiological study of the risk of disease and the relative importance of each mode of transmission was carried out. Cases and controls were selected from a systematic sample of students and teachers present at the school on 28 January. Faecal samples were taken from three food handlers and 16 cases. The influence of each factor was studied using the adjusted odds ratio (aOR) and the estimated population attributable risk (ePAR) with 95\% confidence intervals (CI). We interviewed 210 people (42 cases, 168 controls). The proportion of symptoms in these individuals was nausea $78 \cdot 6 \%$, vomiting $59 \cdot 5 \%$, diarrhoea $45 \cdot 2 \%$, and fever $19 \cdot 0 \%$. The epidemic curve showed transmission for at least 4 days. The risk of disease was associated with exposure to food (aOR $5 \cdot 8$ ) in $66 \cdot 1 \%$ of cases and vomit (aOR $4 \cdot 7$ ) in $24.8 \%$ of cases. Faecal samples from 11 patients and two food handlers were positive for norovirus GII.12 g. Vomit may co-exist with other modes of transmission in norovirus outbreaks and could explain a large number of cases.

Key words: Caliciviruses, epidemiology, Norwalk agent and related viruses, outbreaks, public health.

\section{INTRODUCTION}

Noroviruses have been associated with antigenic changes that lead to regular epidemics [1, 2]. The reservoir and source of norovirus infection are persons with acute disease [3, 4]. Studies in volunteers have revealed totally asymptomatic acute infections that may be an important source of infection $[3,4]$ and

\footnotetext{
* Author for correspondence: Dr P. Godoy, C/Alcalde Rovira Roure 2, 25006 Lleida, Spain.

(Email: pere.godoy@gencat.cat)

$\dagger$ Members of the Working Group are given in the Appendix.
}

could result in chronic carriers [5]. The infectious dose is very low ( $<18$ viral particles) [6] and it is estimated that $1 \mathrm{~g}$ faeces may contain 5 billion infectious doses. Noroviruses are one of the main agents responsible for outbreaks of acute gastroenteritis [7].

Waterborne [8] and foodborne [9-11] transmission have been demonstrated by many observational epidemiological studies [12, 13]. However, person-toperson transmission via surfaces or direct contact with infected persons is more difficult to prove. In norovirus outbreaks, transmission by vomit is an accepted route, but empirical evidence is limited and 
the proof often comes indirectly by ruling out food or water or from descriptive studies [14]. In some outbreaks, these difficulties are even greater because different transmission mechanisms are present simultaneously $[15,16]$. However, as vomiting is a frequent symptom in patients with norovirus gastroenteritis, transmission by vomit (directly or through surface contamination) may explain a large proportion of cases in outbreaks with proximity between cases and susceptible people. Furthermore, vomit could co-exist with other transmission mechanisms that might initiate the outbreak, such as food, water, or contact with a case or contaminated fomites.

In January 2010, an outbreak of gastroenteritis was detected in a high school in Lleida, Spain. In most cases, symptom onset was on the day before notification of the outbreak: some cases had presented with vomiting within the high school and most consumed food in the school dining room, which has its own kitchen.

The aim of this study was to investigate an outbreak of norovirus gastroenteritis in a high school and to assess the relative importance of transmission by food, vomit, or contact with cases.

\section{METHODS}

On 27 January 2010, an outbreak of gastroenteritis that affected students and teachers of different ages and school years was detected in a high school in the city of Lleida, Spain. The school had 573 students aged between 12 and 18 years grouped into six academic years [first, second, third and fourth years of obligatory secondary education (12-16 years) and first and second years of baccalaureate (17-18 years)] and 65 teachers. In many cases, symptoms had begun on 26 January and most cases had consumed food in the school dining room, which has its own kitchen. On the day the outbreak was notified, approximately 150 students had not attended school, mostly due to gastroenteritis, although the school had no register of the causes of absenteeism.

Three food handlers worked in the school kitchen. The head chef presented nausea without vomiting on 24 and 25 January. On Monday 25 January, the chef cooked the day's food but could not finish the workday due to gastrointestinal discomfort. Some affected students had also vomited in different places in the school (restrooms, classrooms, corridors, but not in the dining room). Restrooms were common for the dining room and classes but there was no evidence that sick students used them before consumption of food on 25 and 26 January. The food served was macaroni, sausages with tomatoes, and fruit on 25 January, and soup, roast chicken and fruit and/or yogurt on 26 January. All food was cooked on the same day it was consumed, and was served by the food handlers on metal plates. Students and teachers collected the food and ate it in the dining room next to the kitchen. The school used water from the municipal network and also had a water deposit and a chlorinator. This background led to the hypothesis of an outbreak of gastroenteritis that affected students and teachers caused by consumption of food and/or person-to-person transmission and/or vomit (directly or through surface contamination) from infected persons.

An epidemiological case-control study of the risk associated with eating food in the school, being a close contact of a classmate with gastroenteritis (sitting next to a gastroenteritis case in class) and being exposed to vomit $(<1 \mathrm{~m}$ distance) was made. In addition, the relative importance of each mode of transmission was estimated.

The case definition used was: being a student or teacher at the school who had vomiting and/or diarrhoea or $\geqslant 2$ of the following symptoms: nausea, abdominal pain, or fever, between 23 and 29 January 2010 and who were at school on 28 January when interviews were made. Cases and controls were selected from a systematic sample of the general list of students and teachers.

The three food handlers were interviewed and asked about the handling of food, and were provided with material for the collection of clinical samples. A health inspection of the kitchen was made and water and food samples were taken.

Clinical samples were taken from 16 patients and three food handlers. Samples were pre-screened using standard microbiological tests to rule out bacteria (Salmonella, Shigella, Yersinia, Vibrio, Campylobacter, Aeromonas, verotoxigenic E. coli), parasites, rotaviruses and adenoviruses. Screening for norovirus was performed using two one-step quantitative reverse transcriptase-polymerase chain reaction (qRT-PCR) assays. Samples were analysed using a duplex qRT-PCR assay based on the primers and hydrolysis probes described by Kageyama et al. [17]. Genogroups were assigned after amplification by semi-nested RT-PCR of the ORF1/ORF2 junction region (region $\mathrm{C}$ ) as previously described by Pérez et al. [18]. 
Qualitative variables were expressed using percentages and $95 \%$ confidence intervals (CI) and quantitative variables by calculating the mean and standard deviation (s.D.). The epidemic curve was constructed using 39 cases out of the 42 in which symptom onset could accurately be determined. The number of cases was placed on the y axis and the time of symptom onset on the $\mathrm{x}$ axis. To calculate the days of transmission, we subtracted the minimum incubation period $(24 \mathrm{~h})$ from the first case in the epidemic curve and the maximum incubation period from the last case. Statistical associations were determined using the $\chi^{2}$ test, with the level of statistical significance set at $0 \cdot 05$. The influence of each factor on the risk of disease was studied using the odds ratio (OR) and its 95\% CI. The adjusted ORs (aORs) were calculated using an unconditional logistic regression model including age group, sex and the three factors involved in order to estimate the independent involvement of eating food in the dining room, exposure to vomit (directly or through surface contamination) and being in close contact with cases.

The fraction of the risk of gastroenteritis attributable to each factor in the overall study was estimated using the prevalence of exposure in all those interviewed by estimating the population attributable risk (ePAR):

$\operatorname{ePAR}=(p[(\mathrm{OR}-1)] / p[\mathrm{OR}-1)+1]) \times 100$,

where $p$ is the prevalence of exposure for the respective factor detected in all respondents.

Research ethics committee approval was not required as this was a public health problem regulated by legislation on diseases under public health surveillance.

\section{RESULTS}

Of the 641 people exposed (573 students, 65 teachers, three food handlers), a systematic sample of 210 (42 cases, 168 controls) were interviewed. With respect to controls, cases had a similar proportion of females $(59 \cdot 5 \%$ vs $57 \cdot 7 \%)$ but a slightly lower mean age (14.9 $\pm 6 \cdot 0$ vs. $18 \cdot 3 \pm 10 \cdot 4$ years). On the day the outbreak was notified and the following day student absenteeism was $28 \cdot 4 \%(163 / 573)$ and $25 \cdot 1 \%(144 / 573)$, respectively, and affected all school years (Table 1). Although the school had no specific register of the causes of absenteeism, as the absenteeism baseline in this school was below $1 \%$, we calculated that at least $25 \%$ of students $(143 / 573)$ had not attended classes due to gastroenteritis. The main symptoms were: abdominal pain in $90 \cdot 5 \%(38 / 42)$, nausea in $78 \cdot 6 \%$ $(33 / 42)$, vomiting in $59 \cdot 5 \%(25 / 42)$, diarrhoea in $45 \cdot 2 \%(19 / 42)$ and fever in $19 \cdot 0 \%(8 / 42)$ of cases, and most recovered within $48-72 \mathrm{~h}$. In three cases we were unable to assess the time of onset of symptoms accurately. The epidemic curve, constructed from $39(92.9 \%)$ cases in which the day and time of the first symptom was identified, showed that transmission had been maintained for at least 4 days and was consistent with foodborne and person-to-person transmission (Fig. 1). Six cases presented before the consumption of food on 25 January (one was the chef), the highest number of cases $(20 / 39,51 \cdot 3 \%)$ presented on 26 January after consuming food and the last cases were detected on 28 January.

All food consumed on 25 and 26 January (except fruit on 26 January) was closely associated with the risk of disease (Table 2). Consuming food in the dining room on the 25 and 26 January, a history of contact with a case, and exposure to vomit at a distance of $<1 \mathrm{~m}$ were also associated with the risk of becoming ill (Table 3). In the multivariate logistic regression analysis, consuming food in the dining room (aOR 5.8, $95 \%$ CI $1 \cdot 8-19 \cdot 3$ ) and exposure to vomit (aOR $4 \cdot 7$, $95 \%$ CI $2 \cdot 0-11 \cdot 2$ ) were associated with the risk of becoming ill, while contact with a case was not significant (Table 3). Because exposure to food was greater than exposure to vomit, exposure to food may be stated to have been responsible for a greater number of cases. Thus, according to the ePAR, food consumption would explain $66 \cdot 1 \%(95 \%$ CI $85 \cdot 4-31 \cdot 0)$ of cases and exposure to vomit $24 \cdot 8 \%$ (95\% CI 56.9-3.5).

Bacteriological analysis of water, food and stool cultures from samples of patients and food handlers were negative. In the PCR analysis, 11 faecal samples from patients and two from food handlers tested positive for norovirus genogroup GII.12 g. One positive food handler was the person who cooked the food on 25 and 26 January and the other food handler, who was totally asymptomatic, helped serve the food. Seven days after the first sample, another sample was taken from the two food handlers, which were negative.

\section{DISCUSSION}

This study confirmed a norovirus outbreak that affected students, teachers, and food handlers from the same school and was transmitted by exposure to contaminated food in $66 \cdot 1 \%$ of cases and to vomit 
Table 1. Distribution of absenteeism among senior high-school students and teachers affected by a norovirus outbreak by year and study coverage, Lleida, Spain

\begin{tabular}{lclll}
\hline \hline Students per year, teachers and food handlers & Total & $\begin{array}{l}\text { Students absent } \\
\text { 27 January } n(\%)\end{array}$ & $\begin{array}{l}\text { Students absent } \\
\text { 28 January } n(\%)\end{array}$ & $\begin{array}{l}\text { Interviewed } \\
n(\%)\end{array}$ \\
\hline 1st-year ESO students & 93 & $28(30 \cdot 1)$ & $22(23 \cdot 7)$ & $34(36 \cdot 6)$ \\
2nd-year ESO students & 94 & $30(31 \cdot 9)$ & $29(30 \cdot 9)$ & $30(31 \cdot 9)$ \\
3rd-year ESO students & 110 & $39(35 \cdot 5)$ & $27(24 \cdot 5)$ & $39(35 \cdot 5)$ \\
4th-year ESO students & 89 & $32(36 \cdot 0)$ & $24(27 \cdot 0)$ & $32(36 \cdot 0)$ \\
1st-year baccalaureate students & 92 & $19(20 \cdot 7)$ & $26(28 \cdot 3)$ & $25(27 \cdot 2)$ \\
2nd-year baccalaureate students & 95 & $15(15 \cdot 8)$ & $16(16 \cdot 8)$ & $30(31 \cdot 6)$ \\
Total students & $\mathbf{5 7 3}$ & $\mathbf{1 6 3 ( 2 8 \cdot 4 )}$ & $\mathbf{1 4 4}(\mathbf{2 5} \cdot \mathbf{1})$ & $\mathbf{1 9 0}(\mathbf{3 3} \cdot \mathbf{2})$ \\
Teachers & 65 & & & $17(26 \cdot 2)$ \\
Food handlers & 3 & & & $3(100 \cdot 0)$ \\
Total & $\mathbf{6 4 1}$ & & $\mathbf{2 1 0}(\mathbf{3 2 \cdot 8})$ \\
\hline \hline
\end{tabular}

ESO, Obligatory secondary education (12-16 years).

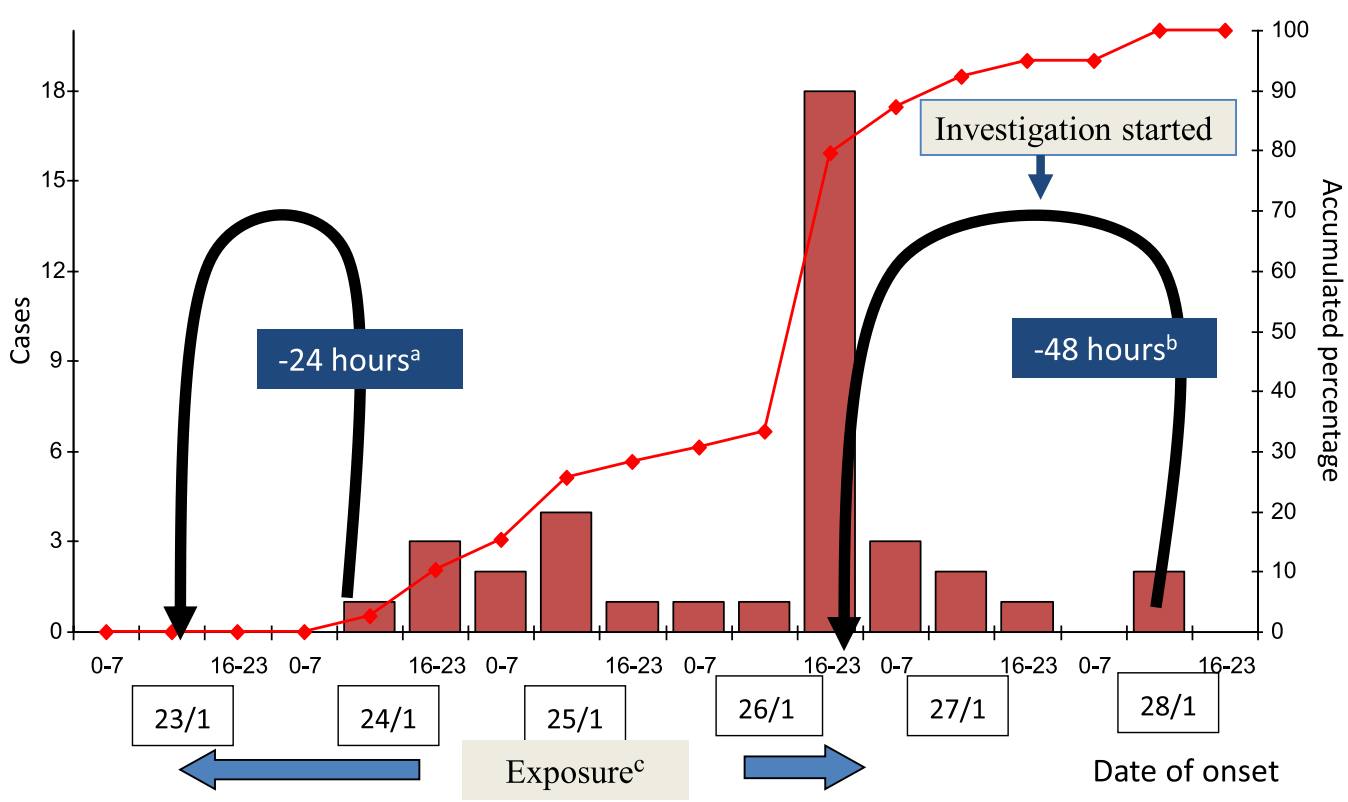

Fig. 1. Epidemic curve of a norovirus outbreak in a high school, Lleida, Spain $(n=39) .{ }^{\text {a }}$ Subtraction of the minimum incubation period $(-24 \mathrm{~h})$ from the first case in the epidemic curve. ${ }^{b}$ Subtraction of the maximum incubation period $(-48$ h) from the last case in the epidemic curve. ${ }^{c}$ To calculate the days of transmission, we determined the time between the dates resulting from subtracting the minimum incubation period from the first case [23 January (23/1)] in the epidemic curve and the maximum incubation period from the last case [26 January (26/1)].

(directly or through surface contamination) in $24.8 \%$ of cases.

The investigation showed the inherent difficulty of outbreaks with multiple transmission routes, when it is particularly difficult to prove both the source and the transmission mechanism itself $[15,16,19,20]$. In addition, person-to-person transmission by contact or from aerosols generated by vomit presents an added difficulty [21-23]. However, the involvement of food was consistent with the epidemic curve, the days of consumption of food cooked by infected food handlers, and the findings of the multivariate analysis of the consumption of food in the school dining room. The involvement of exposure to vomit shown in the multivariate analysis is explained by the episodes of vomiting experienced by many affected students in the school restrooms, classrooms, and corridors. 
Table 2. Analysis of food consumed in the high-school dining room affected by a norovirus outbreak, Lleida, Spain

\begin{tabular}{|c|c|c|c|c|}
\hline Food & $\begin{array}{l}\text { Cases } \\
n(\%)\end{array}$ & $\begin{array}{l}\text { Controls } \\
n(\%)\end{array}$ & OR & $95 \% \mathrm{CI}$ \\
\hline \multicolumn{5}{|c|}{ Macaroni } \\
\hline Yes & $32(76 \cdot 2)$ & $61(36 \cdot 3)$ & $5 \cdot 6$ & \multirow[t]{2}{*}{$2 \cdot 6-12 \cdot 2$} \\
\hline No & $10(23 \cdot 8)$ & $107(63 \cdot 7)$ & 1 & \\
\hline \multicolumn{5}{|c|}{ Sausages } \\
\hline Yes & $32(76 \cdot 2)$ & $60(35 \cdot 7)$ & $5 \cdot 3$ & \multirow{2}{*}{$2 \cdot 6-12 \cdot 5$} \\
\hline No & $10(23 \cdot 8)$ & $108(64 \cdot 3)$ & 1 & \\
\hline \multirow{2}{*}{\multicolumn{5}{|c|}{ Fruit (Monday }} \\
\hline $25 \mathrm{Jar}$ & & & & \multirow{3}{*}{$1 \cdot 6-6 \cdot 4$} \\
\hline Yes & $21(50 \cdot 0)$ & $40(23 \cdot 8)$ & $3 \cdot 2$ & \\
\hline No & $21(50 \cdot 0)$ & $128(76 \cdot 2)$ & 1 & \\
\hline \multicolumn{5}{|l|}{ Soup } \\
\hline Yes & $33(78 \cdot 6)$ & $62(36 \cdot 9)$ & $6 \cdot 3$ & \multirow[t]{2}{*}{$2 \cdot 8-13 \cdot 9$} \\
\hline No & $9(21 \cdot 4)$ & $106(63 \cdot 1)$ & 1 & \\
\hline \multicolumn{5}{|c|}{ Chicken } \\
\hline Yes & $31(73 \cdot 8)$ & $61(36 \cdot 3)$ & $4 \cdot 9$ & \multirow[t]{2}{*}{$2 \cdot 3-10 \cdot 5$} \\
\hline No & $11(26 \cdot 2)$ & $107(63 \cdot 7)$ & 1 & \\
\hline \multicolumn{5}{|c|}{ Yoghurt } \\
\hline Yes & $24(57 \cdot 1)$ & $46(27 \cdot 4)$ & $3 \cdot 5$ & \multirow[t]{2}{*}{$1 \cdot 7-7 \cdot 1$} \\
\hline No & $18(42 \cdot 9)$ & $122(72 \cdot 6)$ & 1 & \\
\hline \multirow{2}{*}{\multicolumn{5}{|c|}{ Fruit (Tuesday }} \\
\hline $26 \mathrm{Jar}$ & & & & \\
\hline Yes & $4(9 \cdot 5)$ & $13(7 \cdot 7)$ & $1 \cdot 2$ & \multirow{2}{*}{$0 \cdot 4-4 \cdot 0$} \\
\hline No & $38(90 \cdot 5)$ & $155(92 \cdot 3)$ & 1 & \\
\hline
\end{tabular}

OR, Odds ratio; CI, confidence interval.

The epidemic curve showed that some cases in students occurred before exposure to food from the canteen, which could be explained by epidemic norovirus activity in the community. This is consistent with the relatively high incidence of norovirus in the population and its periodic emergence [2, 24-28]. The food handler who directly cooked the food presented symptoms compatible with norovirus gastroenteritis 2 days before notification of the outbreak, with norovirus being detected in faeces. Another, completely asymptomatic, food handler who helped serve the food was also positive for norovirus. Conceivably, the symptomatic food handler who cooked the food was the most likely source of contamination [29], but the possible role of the asymptomatic food handler as a source of infection cannot be ruled out. The role of asymptomatic food handlers in outbreaks is unclear: some studies suggest they may present viral loads similar to those of symptomatic food handlers and may be potential sources of the contamination of food $[3,11,29,30]$.

As there were no cases of vomiting in the dining room, the epidemiological analysis found that food consumption in the dining room on 25 and 26 January was the main factor and it may therefore be concluded that transmission occurred mainly due to the food consumed. However, we cannot rule out the possibility that, in some cases, the infection could have been due to some other event in the dining room, for example touching the handle of a ladle used to transfer food to plates. The association found for exposure to vomit in the restrooms, classrooms, and corridors in the multivariate analysis (aOR 4.7) may explain some of the cases who became ill without consuming food in the dining room. Furthermore, the contamination of surfaces, onto which the droplets of vomit containing norovirus could have landed, may have produced some infections. Although the impact of faecal contamination of surfaces may be small compared to contamination of surfaces via vomit, it may, in some cases, be a significant source of contamination [31]. The involvement of vomiting in outbreaks due to the formation of aerosols that can remain in the air for some time and can then be breathed in and swallowed or through contamination of surfaces has been proposed [16, 19, 32]. Thus, exposure to vomit could involve both of these mechanisms. As the infectious dose is extremely low, this route may explain much of the transmission in nonfoodborne, non-waterborne outbreaks. This route has been proposed repeatedly in outbreaks in nursing homes [33], hospitals [34], schools [32] and in travellers [35], where there is close contact between travellers on buses [36], cruise ships [35] and aircraft [17-19]. The involvement of direct contact with cases, especially in centres for dependent persons, such as nursing homes, has also been reported [14]. In the present study, close contact with cases was not significant in the multivariate analysis, suggesting a greater involvement of vomit in groups where personal contacts, such as in schools, are perhaps not so close or continuous [14].

The relative importance of each transmission route in the outbreak is difficult to establish, but given the lack of exposure to vomit in the dining room and the greater exposure to food, this explained a greater number of cases (ePAR 66.1\%) than vomiting (ePAR 24.8\%). Vomit was considered only when exposure was at $<1 \mathrm{~m}$ and therefore this should be considered the minimal involvement. In fact, although increased distance reduces the risk [21], possible exposures at greater distances have also been reported [22]. Taken together, consuming food and exposure to vomit may explain up to $90 \%$ of cases in this 
Table 3. Multivariate analysis of factors involved in a norovirus outbreak in a high school, Lleida, Spain

\begin{tabular}{|c|c|c|c|c|c|c|}
\hline Factor & Cases $n(\%)$ & Controls $n(\%)$ & $\mathrm{cOR}$ & $95 \% \mathrm{CI}$ & aOR & $95 \% \mathrm{CI}$ \\
\hline \multicolumn{7}{|l|}{ Sex } \\
\hline Female & $25(59 \cdot 5)$ & $97(57 \cdot 7)$ & $1 \cdot 1$ & $0 \cdot 5-2 \cdot 1$ & $1 \cdot 1$ & $0 \cdot 5-2 \cdot 5$ \\
\hline Male & $17(40 \cdot 5)$ & $71(42 \cdot 3)$ & 1 & 1 & 1 & \\
\hline \multicolumn{7}{|c|}{ Age group, years } \\
\hline $10-12$ & $8(19 \cdot 0)$ & $25(14 \cdot 9)$ & $6 \cdot 4$ & $0 \cdot 7-55 \cdot 5$ & $5 \cdot 2$ & $0 \cdot 6-47 \cdot 6$ \\
\hline $13-15$ & $26(61 \cdot 9)$ & $63(37 \cdot 5)$ & $8 \cdot 2$ & $1 \cdot 1-64 \cdot 7$ & $8 \cdot 3$ & $1 \cdot 0-67 \cdot 4$ \\
\hline $16-18$ & $7(16 \cdot 7)$ & $60(35 \cdot 7)$ & $2 \cdot 3$ & $0 \cdot 3-20 \cdot 1$ & $5 \cdot 4$ & $0 \cdot 6-51 \cdot 6$ \\
\hline$>18$ & $1(2 \cdot 4)$ & $20(11 \cdot 9)$ & 1 & & 1 & \\
\hline \multicolumn{7}{|c|}{ Close contact with patient } \\
\hline Yes & $18(42 \cdot 9)$ & $28(16 \cdot 7)$ & $3 \cdot 75$ & $1 \cdot 8-7 \cdot 8$ & $1 \cdot 4$ & $0 \cdot 5-1 \cdot 4$ \\
\hline No & $24(57 \cdot 1)$ & $140(83 \cdot 3)$ & 1 & & 1 & \\
\hline \multicolumn{7}{|c|}{ Exposure to vomit } \\
\hline Yes & $12(28 \cdot 6)$ & $7(4 \cdot 2)$ & $9 \cdot 2$ & $3 \cdot 3-25 \cdot 2$ & $4 \cdot 7$ & $2 \cdot 0-11 \cdot 2$ \\
\hline No & $30(71 \cdot 4)$ & $161(95 \cdot 8)$ & 1 & & 1 & \\
\hline \multicolumn{7}{|c|}{ Consumed food in dining room } \\
\hline Yes & $34(80 \cdot 9)$ & $71(42 \cdot 3)$ & 6.9 & $2 \cdot 0-16 \cdot 1$ & $5 \cdot 8$ & $1 \cdot 8-19 \cdot 3$ \\
\hline No & $8(19 \cdot 1)$ & $97(57 \cdot 7)$ & 1 & & 1 & \\
\hline
\end{tabular}

cOR, Crude odds ratio; CI, confidence interval; aOR adjusted odds ratio.

outbreak. The other cases might be explained by exposure to contaminated surfaces. Indeed, some of the cases attributable to exposure to vomit might also be due to contaminated surfaces.

Our results have some implications. As vomiting is a frequent symptom in patients with norovirus gastroenteritis, this study suggests that this transmission mechanism may exist in most norovirus outbreaks. Indeed, its relative importance could be greater if vomiting occurs in spaces such as small rooms in residences or on public transport, due to the small volume of air and proximity between persons. Furthermore, its effect could be amplified by the contamination of surfaces. Therefore, we suggest that vomit should be studied as a mode of transmission in all norovirus outbreaks, as it may co-exist with other modes of transmission and explain a large number of cases.

Most cases recovered within $48-72 \mathrm{~h}$, but the outbreak caused high absenteeism among students $(25.0 \%)$ which involved significant disturbance to academic life, with a loss of school and work hours in teachers, food handlers and students.

The same day the study was announced, consistent control measures were established: handwashing before food consumption and after using the restroom, inspection of the school to ensure the provision of soap and paper for hand drying, the exclusion of all cases from the school, including students, teachers and food handlers until $48 \mathrm{~h}$ after the cessation of symptoms, immediate disinfection of surfaces contaminated by vomit and recommendations on general disinfection of all surfaces with 1:1000 sodium hypochlorite [7, 37]. No further cases were detected in the 3 days following the introduction of these measures and therefore the outbreak was declared over.

The study has some limitations. When participants were selected in the high school there was higher student and teacher absenteeism than usual and it was necessary to make a case-control study as the whole cohort of exposed persons was not available. Thus, the cases selected may not be representative of all cases in the school. The case definition used, based on two specific symptoms (vomiting and diarrhoea), was quite specific but the possibility that some cases could have been caused by other aetiologies cannot be ruled out. Likewise, it has been estimated that up to $30 \%$ of norovirus infections may be asymptomatic $[3,37]$, and therefore some of the controls could really have been cases. This may explain why some controls did not become ill after consuming food in the dining room. However, this would skew the results towards the null hypothesis, which would further strengthen the associations identified in the study. The involvement of vomit was only assessed qualitatively for distances of $<1 \mathrm{~m}$, and therefore the possible involvement of contact at greater distances [21, 22] was not studied. However, some cases (at least 10\%) not explained by consumption of food or exposure to vomit might be due to surface contamination. Indeed, some of the cases attributable to exposure to 
vomit might also be due to contaminated surfaces. Finally, the application of control measures coincided with the closing of the school for the weekend and no comprehensive monitoring was made in families affected, therefore the effectiveness of monitoring could not be documented.

The study has some public health implications. The outbreak occurred in a context in which students and food handlers attended school despite being ill. This facilitated transmission through the consumption of contaminated food and exposure to vomit, making the explosive propagation of norovirus more likely. The outbreak could have been avoided by initial patients not attending school or work. Although the effectiveness of excluding symptomatic food handlers from work is unclear, there is agreement that infected food handlers should be excluded until at least $48 \mathrm{~h}$ after recovery [7, 38]. Samples taken from the two food handlers 7 days after the first sample were negative and they returned to work, although elimination of the virus may take more than 4 weeks and the usefulness of these control samples in food handlers is unknown [7].

In conclusion, vomiting could co-exist with other transmission mechanisms in norovirus outbreaks and may explain a large number of cases. In addition, in schools, high levels of hand hygiene must be maintained, including sufficient soap and paper in restrooms, and sick children and food handlers should be systematically excluded from school, especially if they are suffering from vomiting.

\section{APPENDIX}

\section{Working Group for the Study of Outbreaks of Acute Gastroenteritis in Catalonia}

S. Broner, P. Godoy, A. Martinez, and N. Torner (Public Health Agency of Catalonia, Barcelona, Spain and CIBERESP); M. Alsedà, J. Álvarez, C. Arias, A. Artigues, I. Barrabeig, P. J Balanyà, N. Camps, M. Company, M. Carol, G. Ferrús, N. Follia, S. Minguell, I. Parrón, A. Rovira, M. R. Sala-Farré, R. Torra, J. Torres (Public Health Agency of Catalonia. Barcelona, Spain); M. de Simón, D. Ferrer, A. Moreno, M. Sanz (Public Health Agency of Barcelona, Barcelona, Spain); R. Bartolomé, T. Cornejo (Microbiology Department, University Hospital Vall d'Hebron, Barcelona, Spain); A. Bosch, S. Guix, R. Pintó (Enteric Virus Department, University of Barcelona,
Barcelona, Spain); A. Domínguez (University of Barcelona, Barcelona, Spain and CIBERESP).

\section{ACKNOWLEDGEMENTS}

This study was funded by the National Plan of I+D+I 2008-2011 and ISCIII-Subdirección General de Evaluación y Fomento de la Investigación (Project PI09/02516) and cofunded by Fondo Europeo de Desarroollo Regional (FEDER; Unión Europea, Una manera de hacer Europa), and the Catalan Agency for the Management of Grants for University Research (AGAUR Grant number 2014/ SGR 1403).

\section{DECLARATION OF INTEREST}

None.

\section{REFERENCES}

1. Siebenga JJ, et al. Norovirus illness is a global problem: emergence and spread of norovirus GII.4 variants, 2001-2007. Journal of Infectious Diseases 2009; 200: 802-812.

2. Fonager J, Barzinci S, Fischer T. Emergence of a new recombinant Sydney 2012 norovirus variant in Denmark, 26 December 2012 to 22 March 2013. Eurosurveillance 2013; 18: 20506.

3. Atmar RL, et al. Norwalk virus shedding after experimental human infection. Emerging Infectious Diseases 2008; 14: 1553-1557.

4. Phillips G, et al. Diagnosing norovirus-associated infectious intestinal disease using viral load. BMC Infectious Diseases 2009; 9: 63.

5. Karst SM, Baric RS. What is the reservoir of emergent human norovirus strains? Journal of Virology 2015; 89: $5756-5759$

6. Teunis PF, et al. Norwalk virus: how infectious is it? Journal of Medical Virology 2008; 80: 1468-1476.

7. Centers for Disease Control and Prevention. Updated norovirus outbreak management and disease prevention guidelines. Morbidity and Mortality Weekly Report 2011; 60: 1-18.

8. Centers for Disease Control and Prevention. Surveillance for waterborne disease outbreaks associated with drinking water and other nonrecreational water-United States, 2009-2010. Morbidity and Mortality Weekly Report 2013; 62: 714-720.

9. Matthews JE, et al. The epidemiology of published norovirus outbreaks: a review of risk factors associated with attack rate and genogroup. Epidemiology and Infection 2012; 140: 1161-1172.

10. Dowell SF, et al. A multistate outbreak of oysterassociated gastroenteritis: implications for interstate tracing of contaminated shellfish. Internet Journal of Infectious Diseases 1995; 171: 1497-1503. 
11. Godoy P, et al. Outbreak of food-borne Norovirus associated with the consumption of sandwiches. Medicina Clínica 2005; 124: 161-164.

12. Centers for Disease Control and Prevention. Surveillance for foodborne disease outbreaks - United States, 19982008. Morbidity and Mortality Weekly Report 2013; 62: $1-34$.

13. Hall AJ, et al. foodborne norovirus outbreaks - United States, 2009-2012. Morbidity and Mortality Weekly Report 2014; 63: 491-495.

14. Centers for Disease Control and Prevention. Outbreaks of acute gastroenteritis transmitted by person-to-person contact-United States, 2009-2010. Morbidity and Mortality Weekly Report 2012; 61: 1-12.

15. Thornley CN, et al. Recurring norovirus transmission on an airplane. Clinical Infectious Diseases 2011; 53: 515-520.

16. Lopman B. Air sickness: vomiting and environmental transmission of norovirus on aircraft. Clinical Infectious Diseases 2011; 53: 521-522.

17. Kageyama T, et al. Broadly reactive and highly sensitive assay for Norwalk-like viruses based on real-time quantitative reverse transcription-PCR. Journal of Clinical Microbiology 2003; 41: 1548-1557.

18. Pérez U, et al. Human norovirus occurrence and diversity in the Llobregat river catchment, Spain. Environmental Microbiology 2012; 14: 494-502.

19. Kirking HL, et al. Likely transmission of norovirus on an airplane, October 2008. Clinical Infectious Diseases 2010; 50: 1216-1221.

20. Becker KM, et al. Transmission of Norwalk virus during football game. New England Journal of Medicine 2000; 343: 1223-1227.

21. Booth CM. Vomiting Larry: a simulated vomiting system for projectile vomiting related to norovirus infection. Journal of Infection Prevention 2014; 15: 176-180.

22. Marks PJ, et al. Evidence for airborne transmission of Norwalk-like virus (NLV) in a hotel restaurant. Epidemiology and Infection 2000; 124: 481-487.

23. Widdowson MA, et al. Probable transmission of norovirus on an airplane. JAMA Internal Medicine 2005; 293: $1859-1860$.

24. Hall AJ, et al. Norovirus disease in the United States. Emerging Infectious Diseases 2013; 19: 1198-1205.

25. Ahmed SM, et al. Global prevalence of norovirus in cases of gastroenteritis: a systematic review and meta-analysis. Lancet. Infectious Diseases 2014; 14: 725-730.

26. Gibney KB, et al. Disease burden of selected gastrointestinal pathogens in Australia, 2010. International Journal of Infectious Diseases 2014; 28: 176-185.

27. Patel MM, et al. Systematic literature review of role of noroviruses in sporadic gastroenteritis. Emerging Infectious Diseases 2008; 14: 1224-1231.

28. Arena C, et al. Acute diarrhea in adults consulting a general practitioner in France during winter: incidence, clinical characteristics, management and risk factors. BMC Infectious Diseases 2014; 14: 574.

29. Franck KT, et al. Sources of calicivirus contamination in foodborne outbreaks in Denmark, 2005-2011 - the role of the asymptomatic food handler. Journal of Infectious Diseases 2015; 211: 653-670.

30. Barrabeig I, et al. Foodborne norovirus outbreak: the role of an asymptomatic food handler. $B M C$ Infectious Diseases 2010; 10: 1-7.

31. Repp KK, Hostetler TP, Keene WE. A norovirus outbreak related to contaminated surfaces. Journal of Infectious Diseases 2013; 208: 295-298.

32. Marks PJ, et al. A school outbreak of Norwalk-like virus: evidence for airborne transmission. Epidemiology and Infection 2003; 131: 727-736.

33. Godoy $\mathbf{P}$, et al. Norovirus gastroenteritis outbreak by person-to-person transmission in a nursing home. Medicina Clínica 2006; 127: 538-541.

34. Harris JP, et al. Does spatial proximity drive norovirus transmission during outbreaks in hospitals? BMJ Open 2013; 3: 1-6.

35. Wikswo ME, et al. Disease transmission and passenger behaviors during a high morbidity Norovirus outbreak on a cruise ship, January 2009. Clinical Infectious Diseases 2011; 52: 1116-1122.

36. Solano R, et al. Person-to-person transmission of norovirus resulting in an outbreak of acute gastroenteritis at a summer camp. European Journal of Gastroenterology \& Hepatology 2014; 26: 1160-1166.

37. Greig JD, Lee MB. A review of nosocomial norovirus outbreaks: infection control interventions found effective. Epidemiology and Infection 2012; 140: 1151-1160.

38. Harris JP, Lopman BA, O'Brien SJ. Infection control measures for norovirus: a systematic review of outbreaks in semi-enclosed settings. Journal of Hospital Infection 2010; 74: 1-9. 